\title{
ERRATUM
}

Sakhr A. Murshid • Hiroshi Kamioka • Yoshihito Ishihara

Ryoko Ando • Yasuyo Sugawara

Teruko Takano-Yamamoto

\section{Actin and microtubule cytoskeletons of the processes of 3D-cultured MC3T3-E1 cells and osteocytes}

\section{J Bone Miner Metab (2007) 25:151-158}

DOI 10.1007/s00774-006-0745-5

The authors' affiliations appeared incorrectly in the article cited above. The correct authors' affiliations are as follows.

T. Takano-Yamamoto $(\bowtie)$

Division of Orthodontics and Dentofacial Orthopedics, Graduate School of Dentistry, Tohoku University,

4-1 Seiryo-machi, Aoba-ku, Sendai 980-8574, Japan

Tel./Fax +81-22-717-8372

e-mail: t-yamamo@mail.tains.tohoku.ac.jp

S.A. Murshid · H. Kamioka · Y. Ishihara · R. Ando •

Y. Sugawara

Department of Orthodontics and Dentofacial

Orthopedics, Okayama University Graduate School of Medicine, Dentistry, and Pharmaceutical Sciences,

Okayama, Japan 